\title{
Dynamical Heterogeneity and Nonlinear Susceptibility in Supercooled Liquids with Short-Range Attraction
}

\author{
P. Charbonneau ${ }^{1, *}$ and D. R. Reichman ${ }^{2}$ \\ ${ }^{1}$ Chemistry and Chemical Biology, Harvard University, 12 Oxford Street, Cambridge, Massachusetts 02138, USA \\ ${ }^{2}$ Department of Chemistry, Columbia University, 3000 Broadway, New York, New York 10027, USA
}

(Received 12 June 2007; published 24 September 2007)

\begin{abstract}
Recent work has demonstrated the strong qualitative differences between the dynamics near a glass transition driven by short-ranged repulsion and one governed by short-ranged attraction. Here we study in detail the behavior of nonlinear, higher-order correlation functions that measure the growth of length scales associated with dynamical heterogeneity in both types of systems. We find that this measure is qualitatively different in the repulsive and attractive cases with regards to the wave vector dependence as well as the time dependence of the standard nonlinear four-point dynamical susceptibility. We discuss the implications of these results for the general understanding of dynamical heterogeneity in glass-forming liquids.
\end{abstract}

DOI: 10.1103/PhysRevLett.99.135701

PACS numbers: 64.70.Pf, 61.43.Fs, 82.70.Dd

The underlying reasons for the dramatic increase in the viscosity of glass-forming liquids are not well understood. It has become increasingly clear that simple structural measures remain short-ranged close to the glass transition, and thus a growing simple static length scale does not appear to be implicated [1]. This has led to the search for a growing dynamical length scale that drives vitrification. Indeed, recent simulations [2-7] and experiments [8-13] have given direct evidence for both a growing length scale and a dynamical scaling relating its growth to the rapidly increasing time scales that characterize the glass transition. The study of this key aspect of dynamical heterogeneity, as encoded in various multipoint dynamical susceptibilities, has opened up the ability both to extract absolute length scales associated with cooperative relaxation in glassy systems and to provide precise metrics for the testing of various theoretical approaches $[5,14,15]$.

The simplest model system that exhibits the expected dynamical behavior associated with more complicated glassy systems is the hard-sphere liquid. Here entropydriven crowding effects give rise to a characteristic dynamical behavior that includes a two-step nonexponential relaxation, a dramatic increase in relaxation times associated with small changes in volume fraction, and dynamical heterogeneity accompanied by a growing dynamical length scale [14]. Recently, it has been demonstrated via theory [16], simulation $[17,18]$, and experiment $[19,20]$ that another extreme glassy limit exists for simple spherical particles: that of the short-range attractive glassy state. Here strong short-ranged bonding between the particles can lead to extremely slow relaxation but with dramatically different dynamical characteristics. Dynamical heterogeneity also exists close to the attractive glassy state [17,21-23] but has not been systematically characterized, and multipoint dynamical susceptibilities for such systems have not been measured or computed. The goal of this work is to investigate in detail the properties of standard nonlinear spatiotemporal susceptibilities at distinct points along the attractive glass line and to quantitatively and qualitatively compare the observed features to those of the hard-sphere system that lies at the infinite temperature limit of the dynamical arrest line. The outcome of this exercise is a greater understanding of the physics of attractive glassforming systems and dynamical heterogeneity in glassforming liquids in general, as well as important benchmarks for the testing for theoretical treatments.

The systems we consider have potentials of the form

$$
U(r)=4 \varepsilon\left[\left(\frac{\sigma_{\alpha \beta}}{r}\right)^{2 n}-\left(\frac{\sigma_{\alpha \beta}}{r}\right)^{n}\right],
$$

where the temperature scale $T$ is set by $\varepsilon$ and the length scale by $\sigma_{B B}$, so time $t$ is rescaled by $\left(\varepsilon / m \sigma_{A A}^{2}\right)^{1 / 2}$. For our study, $n=40$ and 30, yielding a potential with an attractive range of approximately $3 \%$ and $4 \%$ of $\sigma_{B B}$, respectively. To prevent crystallization, a 50:50 mixture with size ratio $\sigma_{A A} / \sigma_{B B}=1.2$ and $\sigma_{A B}=\sigma_{B A}=\left(\sigma_{A A}+\sigma_{B B}\right) / 2$ is used. Standard molecular dynamics (MD) simulations with a number of particles $N=256$ have been performed in the microcanonical ensemble using a time step $\Delta t<$ $1.3 \times 10^{-3}$. Comparison to a $N=2048$ system for finitesize effects found little discernible difference for the quantities studied here. In Fig. 1, we plot a $T$-volume fraction $\phi$ dynamical phase diagram of the system. The arrest line is determined by extrapolation of the isodiffusion curves to the limit of zero diffusivity [24]. Three $(T, \phi, n)$ state points in the supercooled-liquid regime are considered: $A=(4.4,0.605,30), \quad B=(0.36,0.59,30), \quad$ and $\quad C=$ $(0.34,0.6,40)$. Point $A$ lies close to the hard-sphere limit, while point $C$ lies close to the attraction-driven arrest line but away from the putative $A_{3}$ dynamical singularity predicted by mode-coupling theory (MCT) [25]. Point $B$ lies close to the arrest line in the "reentrant pocket," near the location of the higher-order $A_{4}$ singularity predicted by 


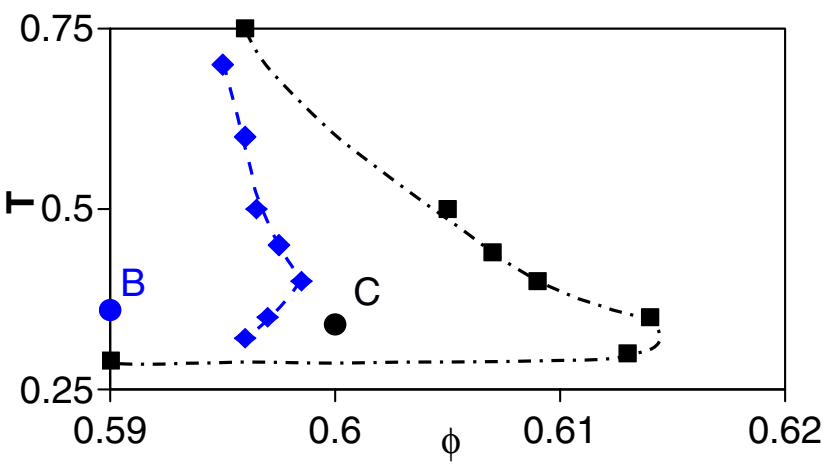

FIG. 1 (color online). Dynamical phase diagram for interaction ranges $n=30$ (diamonds) and $n=40$ (squares) obtained by extrapolating to the limit of zero diffusivity [24]. The putative higher-order $\left(A_{4}\right)$ singularity lies near the $n=30$ cusp. Simulation points $B$ and $C$ are indicated, while point $A$ lies off the plot in the high- $T$ limit of the $T-\phi$ diagram. The dashed lines are guides for the eye.

MCT. In all cases, state points have been chosen not only to reflect potentially distinct physics but so that the $\alpha$-relaxation times $\tau_{\alpha}$ will be similar as well [26].

Simple two-point dynamical correlation functions have already been extensively characterized in these systems [24]. The two-point function $F_{s}(k, t)$ displays drastically different decay characteristics at the three selected state points, ranging from standard two-step relaxation with associated power-law relaxation at point $A$ to intermediatetime logarithmic decay spanning several time decades at point $B$. Here we characterize the fluctuations of two-point dynamical quantities that are directly relevant for understanding dynamical heterogeneity. We focus, in particular, on the normalized susceptibility

$$
\chi_{4}(k, t)=\frac{\left\langle f_{s}(k, t)^{2}\right\rangle-\left\langle f_{s}(k, t)\right\rangle^{2}}{N^{-1} \sum_{i}\left[\left\langle f_{s}^{i}(k, t)^{2}\right\rangle-\left\langle f_{s}^{i}(k, t)\right\rangle^{2}\right]}
$$

where $\quad f_{s}(q, t)=N^{-1} \sum_{i} \cos \left\{\mathbf{k} \cdot\left[\mathbf{r}_{i}(t)-\mathbf{r}_{i}(0)\right]\right\} \equiv$ $N^{-1} \sum_{i} f_{s}^{i}(k, t)$. This quantity measures the size of fluctuations in self-density correlations at a particular wave vector; its growth is related to the increase of a cooperative dynamical length scale. Here, unlike in some earlier work, we explicitly label the wave vector dependence of this susceptibility, which indicates that the fluctuations associated with dynamical heterogeneity may be large or small depending on the intrinsic length scale that is probed. Indeed, as discussed by Chandler et al. [15] (see also Refs. [27,28]), the $k$ dependence of $\chi_{4}(k, t)$ is a useful way to probe the various length scales associated with dynamical heterogeneity. The $k$ dependence of $\chi_{4}(k, t)$ should not be confused with that of $S_{4}(k, t)$, which provides a four-point analog to the static structure factor and allows for the direct extraction of a length scale associated with cooperative heterogeneous motion. On the other hand, it is expected that $\max _{t}\left[\chi_{4}(k, t)\right] \sim \xi(t)^{2-\eta}$, where $\xi(t)$ is the same dynamical heterogeneity length scale extracted from
$S_{4}(k, t)$ [29] and $\eta$ is the susceptibility exponent. Thus, as long as $\eta$ does not vary for the region of interest in the dynamical phase diagram, one may infer some information concerning the growth and absolute size of $\xi(t)$ [30]. Last, it should be noted that the definition of $\chi_{4}(k, t)$ given above slightly differs from the standard definition, due to the normalization factor in the denominator. This normalization is used to attempt an unbiased comparison of peak amplitudes. We have checked that the conclusions drawn from the results presented below are not altered if the standard, unnormalized definition is used.

We start with a comparison between the $k$ dependence of the maximal values of $\chi_{4}(k, t)$ for state points $A$ and $C$. As shown in Fig. 2, a striking qualitative distinction exists between the size of dynamical fluctuations in the cases where glassy behavior is driven by strong, short-ranged bonding compared to the hard-sphere limit, where crowding drives vitrification. In particular, dynamical fluctuations are maximized for wave vectors below that of the main diffraction peak of $S(k)$ in the hard-sphere limit, while in the attraction-driven case the maximal fluctuations occur for wave vectors in excess of the first-neighbor peak of $S(k)$. This makes clear the fact that, while in the hardsphere case dynamical heterogeneity fluctuations are most sensitive to collective events on scales larger than the particle size, in the case of strong short-ranged attractions it is bonding fluctuations that trigger the emergence of dynamical heterogeneity, as measured in $\chi_{4}(k, t)$.

It is instructive to compare this result with the recent calculations of Greenall et al. [33]. In that work, the sensitivity of the $k$-dependent plateau height to changes in the structure, as computed by MCT, was measured for both the hard-sphere and the attractive glass-forming limits. For the hard-sphere system, it was found that sensitivity is most pronounced for changes in structure just beyond the first shell of neighbors. Greenall et al. deemed this the "caged-cage" effect. On the other hand, it was found that

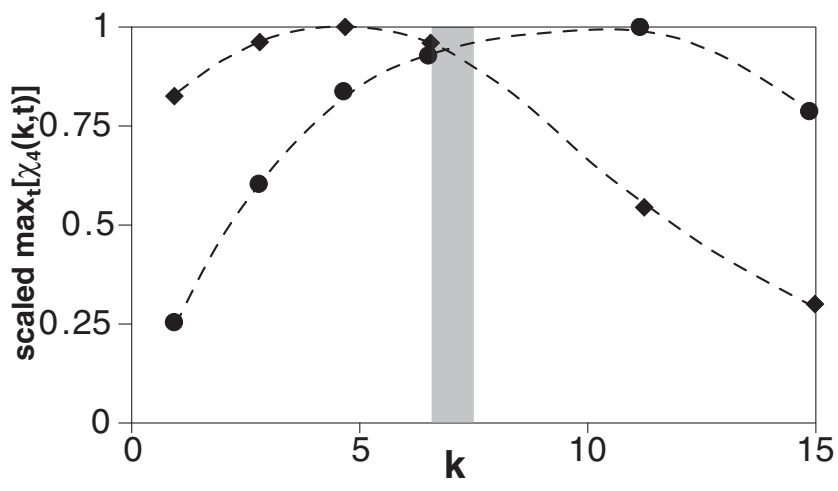

FIG. 2. Maximal values of $\chi_{4}(k, t)$ as a function of wave vector for points $A$ (diamonds) and $C$ (circles), relative to the absolute maximum of $\chi_{4}(k, t)$ for that state point. The $k$ dependence of point $B$ is qualitatively similar to that of point $C$ and is not shown. The nearest-neighbor peak of $S(k)$ for both state points is contained within the shaded area. 
the plateau for systems near the attraction-driven arrest line is most sensitive to changes of structure that occur at high wave vectors associated with short-ranged bonding - i.e., at $k$ values much in excess of an inverse particle size. A strong qualitative similarity is thus seen between the $k$ dependence of the plateau sensitivity computed by MCT and the $k$ dependence in the peak height of $\chi_{4}(k, t)$ measured directly via MD simulation. To interpret this, we first remark that, at least in the $\beta$-relaxation regime, $\chi_{4}(k, t)$ is an indicator of the spatial fluctuations of the plateau height, which has been shown in simulations to be spatially heterogeneous even at these short time scales [34]. Clearly, the MCT calculations measure the sensitivity of the plateau of a uniform system to uniform changes in structure, while at any instant in a real liquid the local structure varies from site to site. However, it is reasonable to assume that these spatial fluctuations will mirror the very same sensitivity to local structure as the global plateau does to a global change in structure. Thus, our dynamical results for the $k$ dependence of the peak height of fluctuations associated with dynamical heterogeneity provide a deep connection with the static MCT calculations of Greenall et al. This interpretation is in harmony with recent calculations and speculations concerning the nature and interpretation of dynamical heterogeneity within MCT $[35,36]$.

We now turn to the full time dependence of $\chi_{4}(k, t)$. In Fig. 3, we show $\chi_{4}(k, t)$ for $k$ values above and below the peak of $S(k)$ for the three different state points. Clearly, the temporal shape of $\chi_{4}(k, t)$ is qualitatively different at the three points. The time-dependent growth of $\chi_{4}(k, t)$ to its peak in the repulsion-driven limit of point $A$ may be fit to a power-law form, as shown in the log-log inset in Fig. 3 (top). This behavior is similar to that observed in many other systems, such as mixtures of soft-sphere or LennardJones particles [5]. This is in contrast to the case of point $B$, which lies closest to a putative MCT higher-order singularity. Here, as shown in Fig. 3 (middle), the amplitude of $\chi_{4}(k, t)$ in the $\beta$ regime is extremely small, while the peak heights in the $\alpha$ regime are sizable and actually exceed those calculated in the hard-sphere case. Thus, the $\beta$ regime at this state point is local in its physics, displaying none of the hallmarks of cooperativity that have already set in at short times in the hard-sphere case. Further, the dramatic change in temporal behavior of $\chi_{4}(k, t)$ from intermediate to long times suggests the possibility of different length scales governing the $\beta$ and $\alpha$ regimes, respectively, in contrast to what is usually observed in typical glassy systems $[31,32]$. Last, at state point $C$, where attractions dominate relaxation but far from the location of the reentrant elbow of the arrest line, a behavior with mixed properties is seen. In particular, the growth of $\chi_{4}(k, t)$ does not display a drastic difference between the $\beta$ and $\alpha$ regimes, although the growth is quite slow, and peak values reach only modest amplitudes.

A close inspection of the behavior of $\chi_{4}(k, t)$ at state point $B$ indicates that it grows in the $\beta$ regime not as a power law but essentially logarithmically in time [see

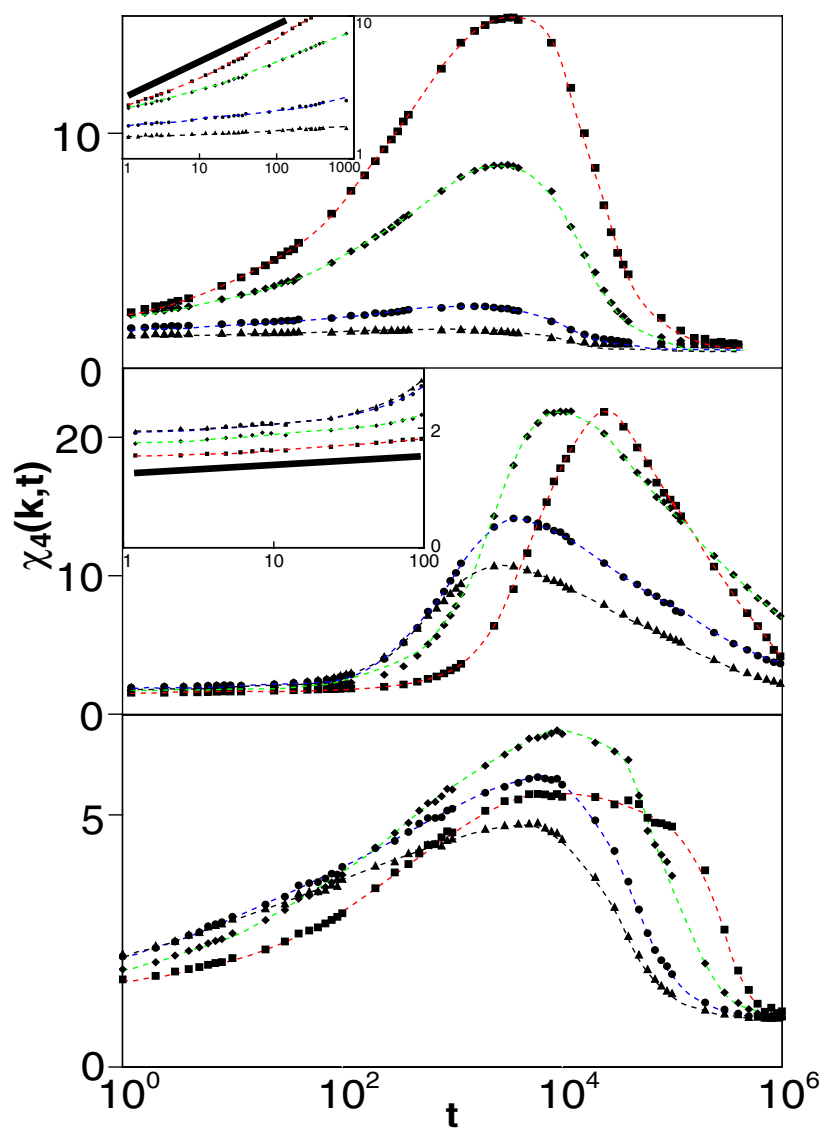

FIG. 3 (color online). Time evolution of $\chi_{4}(k, t)$ for wave vector $k=6.6$ (squares), 11.2 (diamonds), 18.7 (circles), and 22.5 (triangles) at state points $A, B$, and $C$, from top to bottom. Insets: Thick black lines show approximate (top) power-law and (middle) logarithmic growth at intermediate times.

linear-log inset in Fig. 3 (middle)]. While the decay of the two-point function $F_{s}(k, t)$ is known to be nearly logarithmic in this regime [24], it is not at all obvious that this should also be true for its fluctuations. It has recently been argued that the susceptibility $\chi_{T}(k, t) \sim d F_{s}(k, t) / d T$ may serve as a mimic of $\chi_{4}(k, t)$ [14,31,32]. By considering $F_{s}(k, t)$, it is clear that the growth of $\chi_{4}(k, t)$ to its peak should be a power law for standard repulsive systems. On the other hand, the same considerations are not informative for the attractive regime, where the leading term of $\chi_{T}(k, t)$ in the $\beta$ regime is not dependent on time at all. Indeed, argumentation based on the susceptibility $\chi_{T}(k, t)$ would suggest that $\chi_{4}(k, t)$ grows logarithmically in time only if subleading terms in the expansion of the two-point function are a power series in the logarithm, as suggested from MCT analysis near higher-order singularities [25]. Indeed, the result presented here for $\chi_{4}(k, t)$ may be taken as indirect evidence for the reality of these subleading terms.

In conclusion, we have systematically studied how the dynamical heterogeneity indicator $\chi_{4}(k, t)$ varies along the arrest line in attractive colloidal systems. The behavior of $\chi_{4}(k, t)$ in the attraction-dominated limit markedly differs 
from that previously observed in standard repulsiondominated systems. First, the scale of fluctuations is maximized for intrinsic length scales significantly smaller than a particle diameter, as opposed to the hard-sphere case, where fluctuations are maximized at length scales in excess of the particle size. This result suggests that short-ranged bonding fluctuations trigger dynamical heterogeneity in attractive systems, while intrinsic dynamics at scales larger than the cage scale couple most strongly to dynamical heterogeneity in repulsive systems. In addition, the time dependence of $\chi_{4}(k, t)$ varies dramatically from one limit to the other. These suggest marked differences in the degree of cooperativity seen in attractive and repulsive cases. In particular, the amplitude of $\chi_{4}(k, t)$ is much smaller in the $\beta$ regime in the attractive case, and the growth of $\chi_{4}(k, t)$ is logarithmically slow near the onset of reentrance, as opposed to the more common power-law behavior. These results deepen our understanding of the physics of dynamical heterogeneity as well as provide testable targets for theoretical approaches. In particular, it would be interesting to apply the recent extension of MCT of Ref. [35], which has successfully predicted the behavior of $\chi_{4}(k, t)$ and the growth of the dynamical length scale in standard systems to the case of attractive glass-forming systems.

This work was supported in part by Grants No. NSF0134969 and No. FQRNT-91389 (to P. C.). We thank G. Biroli, M.E. Cates, J.D. Eaves, and K. Miyazaki for helpful discussions.

*Present address: FOM Institute AMOLF, Kruislaan 407, 1098 SJ Amsterdam, The Netherlands.

[1] M. D. Ediger, C. A. Angell, and S. R. Nagel, J. Chem. Phys. 100, 13200 (1996).

[2] R. Yamamoto and A. Onuki, Phys. Rev. Lett. 81, 4915 (1998).

[3] C. Donati, J. F. Douglas, W. Kob, S. J. Plimpton, P. H. Poole, and S. C. Glotzer, Phys. Rev. Lett. 80, 2338 (1998).

[4] C. Donati, S. C. Glotzer, P. H. Poole, W. Kob, and S. J. Plimpton, Phys. Rev. E 60, 3107 (1999).

[5] C. Toninelli, M. Wyart, L. Berthier, G. Biroli, and J.-P. Bouchaud, Phys. Rev. E 71, 041505 (2005).

[6] A. Widmer-Cooper and P. Harrowell, J. Phys. Condens. Matter 17, S4025 (2005).

[7] A. Widmer-Cooper, P. Harrowell, and H. Fynewever, Phys. Rev. Lett. 93, 135701 (2004).

[8] M.T. Cicerone, F. R. Blackburn, and M.D. Ediger, Macromolecules 28, 8224 (1995).

[9] R. Böhmer et al., J. Non-Cryst. Solids 235, 1 (1998).

[10] E. V. Russell and N.E. Israeloff, Nature (London) 408, 695 (2000)

[11] E. R. Weeks, J. C. Crocker, A. C. Levitt, A. Schofield, and D. A. Weitz, Science 287, 627 (2000).

[12] R. Richert, J. Phys. Condens. Matter 14, R703 (2002).
[13] A. S. Keys, A. R. Abate, S.C. Glotzer, and D. J. Durian, Nature Phys. 3, 260 (2007).

[14] L. Berthier, G. Biroli, J.-P. Bouchaud, L. Cipelletti, D. El Masri, D. L'Hôte, F. Ladieu, and M. Perino, Science 310, 1797 (2005).

[15] D. Chandler, J. P. Garrahan, R. L. Jack, L. Maibaum, and A. C. Pan, Phys. Rev. E 74, 051501 (2006).

[16] K. Dawson, G. Foffi, M. Fuchs, W. Gotze, F. Sciortino, M. Sperl, P. Tartaglia, T. Voigtmann, and E. Zaccarelli, Phys. Rev. E 63, 011401 (2000).

[17] A. M. Puertas, M. Fuchs, and M. E. Cates, Phys. Rev. E 67, 031406 (2003).

[18] E. Zaccarelli, G. Foffi, K. A. Dawson, S. V. Buldyrev, F. Sciortino, and P. Tartaglia, Phys. Rev. E 66, 041402 (2002).

[19] S. H. Chen, W. R. Chen, and F. Mallamace, Science 300, 619 (2003).

[20] K. N. Pham, A. M. Puertas, J. Bergenholtz, S. U. Egelhaaf, A. Moussaid, P. N. Pusey, A. B. Schofield, M.E. Cates, M. Fuchs, and W. C. K. Poon, Science 296, 104 (2002).

[21] A. M. Puertas, M. Fuchs, and M. E. Cates, J. Chem. Phys. 121, 2813 (2004).

[22] A. M. Puertas, M. Fuchs, and M. E. Cates, J. Phys. Chem. B 109, 6666 (2005).

[23] D. R. Reichman, E. Rabani, and P. L. Geissler, J. Phys. Chem. B 109, 14654 (2005).

[24] F. Sciortino, P. Tartaglia, and E. Zaccarelli, Phys. Rev. Lett. 91, 268301 (2003).

[25] W. Götze and M. Sperl, Phys. Rev. E 66, 011405 (2002).

[26] The relaxation time $\tau_{\alpha}$ is defined as the time at which the self-intermediate scattering function $F_{s}(k, t)=$ $\left\langle N^{-1} \sum_{i} e^{i \mathbf{k} \cdot\left[\mathbf{r}_{i}(t)-\mathbf{r}_{i}(0)\right]}\right\rangle$ has decayed to $e^{-1}$ at the peak of the structure factor $S(k)=\left\langle N^{-1} \sum_{i j} e^{i \mathbf{k} \cdot\left(\mathbf{r}_{j}-\mathbf{r}_{i}\right)}\right\rangle$, where $\mathbf{r}_{i}$ is the position of particle $i$ and $k$ is the magnitude of the wave vector probed.

[27] O. Dauchot, G. Marty, and G. Biroli, Phys. Rev. Lett. 95, 265701 (2005).

[28] V. Trappe, E. Pitard, L. Ramos, A. Robert, H. Bissig, and L. Cipelletti, arXiv:cond-mat/0702646v2.

[29] N. Lačević, F. W. Starr, T. B. Schrøder, and S. C. Glotzer, J. Chem. Phys. 119, 7372 (2003).

[30] This is the case only if $\chi_{4}(k, t)$ is computed in the thermodynamic ensemble consistent with experimental conditions $[14,31,32]$.

[31] L. Berthier, G. Biroli, J.-P. Bouchaud, W. Kob, K. Miyazaki, and D. R. Reichman, J. Chem. Phys. 126, 184503 (2007).

[32] L. Berthier, G. Biroli, J.-P. Bouchaud, W. Kob, K. Miyazaki, and D. R. Reichman, J. Chem. Phys. 126, 184504 (2007).

[33] M. J. Greenall, T. Voigtmann, P. Monthoux, and M.E. Cates, Phys. Rev. E 73, 050501(R) (2006).

[34] A. Widmer-Cooper and P. Harrowell, Phys. Rev. Lett. 96, 185701 (2006).

[35] G. Biroli, J.-P. Bouchaud, K. Miyazaki, and D. R. Reichman, Phys. Rev. Lett. 97, 195701 (2006).

[36] G. Biroli and J.-P. Bouchaud, J. Phys. Condens. Matter 19, 205101 (2007). 